\title{
THE PROBLEM OF THE NATURE AND OF THE SIGNIFICANCE OF THE MITSUDA REACTION TO LEPROMIN
}

\author{
R. Chaussinand \\ (Institut Pasteur, Paris; this paper is reprinted in English translation \\ approved by the author, by kind permission of the Editor, Ann. de \\ I'Institut Pasteur. The article appeared August, 1959, 97, pp. 125-134)
}

The interpretation of the nature and the significance of the reaction to lepromin becomes more and more difficult.

Lepromin is a filtered and autoclaved suspension of I g. of lepromatous nodules, finely ground, in $30 \mathrm{ml}$. of physiological saline, $0.5 \%$ carbolised. This antigen contains Hansen's bacilli heatkilled and some tissue debris. The Mitsuda reaction is found by injecting $0.1 \mathrm{ml}$. of lepromin intradermally. The result is positive when an erythematous infiltration, which reaches its height at the end of 3 to 4 weeks, forms at the point of the injection. Small infiltrations of less than $3 \mathrm{~mm}$. in diameter are considered as doubtful. In strongly positive cases, the nodular infiltration can ulcerate. Towards the 30 th day the histological aspect of the reaction is tuberculoid in type.

Twenty years ago it was considered that sensitivity to lepromin was always a sign of a relative state of immunity against leprosy. This opinion was based on the following premises:

Patients with tuberculoid leprosy, with few bacilli, usually react strongly to the Mitsuda reaction, while leprosy patients of the malignant lepromatous kind, whose body is rapidly invaded by Hansen's bacilli, are insensitive to lepromin.

Moreover, benign leprosy never changes to the malignant type, so long as the patient reacts to lepromin.

Finally, epidemiological enquiries seem to show that those with a positive Mitsuda reaction contract leprosy in contact with contagious patients less frequently than those insensitive to lepromin.

It is admitted then quite reasonably that sensitivity to lepromin was provoked by the impregnation or by the infection of the body by the Hansen's bacillus and that it was witness of the presence of a relative state of immunity, shown in a marked resistance of the tissues to the multiplication and invasion of the pathogenic agent.

Actually, sensitivity to lepromin, owing to the attack on the body by the leprosy bacillus, keeps without any doubt all its prognostic value; clinical and immunological studies amply show this. A positive Mitsuda reaction, determined by a primary leprosy infection, remains the sign of an immuno-allergic state which is expressed in a resistance of the body not only to superinfections but also to the primary infection, by arresting evolution to the malignant type. Immunoallergy in leprosy is therefore different from that observed in 
tuberculosis which protects the body against further infections, but has no action on the evolution of the primary infection.

The immuno-allergic state in leprosy also shows other special features. Thus in a country where leprosy is strongly endemic the Mitsuda reaction is rarely positive in the first two years of life. It is only from the age of three that the number of allergic subjects gradually increases with age. Souza Campos seeks to explain this by a theory based on the existence of a rare, infantile form of tuberculoid leprosy which would cure itself completely without the help of any therapy after the ulceration and scarring of the cutaneous lesions ${ }^{15}$. This author thinks it is possible that the child would benefit from an immunity transmitted by its mother during intra-uterine life.

We have never had the opportunity of observing cases of this early abortive leprosy, but the study of the cases published by Souza Lima, Souza Campos, and Pessôa Mendes ${ }^{16},{ }^{14}$ confirms that the majority of children suffering from this special kind of tuberculoid leprosy have lepromatous mothers. Also it seems improbable to us that these mothers whose bodies show no resistance to the invasion of the leprosy bacillus should be able to transmit to their children a relative immunity which they themselves do not have. We consider moreover that the rarity of sensitivity to lepromin in children of less than 3 years could be due in part to the long incubation of leprosy which seems to be on an average of 2 to 3 years.

Another point is that this immuno-allergic state which can be discovered by the Mitsuda reaction is often varying in its intensity and can even disappear. At the time when chaulmoogra oil was the only remedy for leprosy we were able to note on many occasions that patients with benign leprosy who reacted at first, often strongly, to lepromin would become anergic. (At the present time, the treatment by sulphones, if it is regularly continued, prevents always the malignant evolution and that even in the originally anergic leprosy patients.) The appearance of this insensitivity to lepromin did not show itself in a weakening of the general condition nor in a clinical deterioration (sometimes the cutaneous and neuritic lesions improved) nor even in an increase in the number of bacilli in the lesions. It was only later, at the end of several months and sometimes after many years, that this benign anergic leprosy changed quickly into malignant leprosy. The anergy then is not caused by a strong increase in bacilli in the lesions as some authors claim, as it precedes the invasion of the body by the pathogenic agent. Immuno-allergy in leprosy does not depend on the kind of infection, on the contrary it is the factor that determines the benign or malignant development of the disease. This confirmation is extremely interesting since it provides an important proof of the immunological and prognostic value of the Mitsuda test.

Rotberg ${ }^{17}$ believes that sensitivity to lepromin can only be 
produced in a body with a special power of resistance, the natural $\mathrm{N}$ factor. This factor in the presence of leprosy or tuberculosis infection or of BCG vaccination would bring about sensitivity to lepromin, while organisms without this $\mathrm{N}$ factor would be incapable of reacting to this antigen.

It is futile to say that factors which bring about the appearance and disappearance of allergy in leprosy are not known. Yet, every body has the innate faculty to react to infections, but seemingly in a variable manner according to the race, the family, the age, the sex, and the physiological individuality. In leprosy, anergy does not seem to depend on the absence of a hypothetical $\mathrm{N}$ factor, but rather on metabolic phenomena capable of depriving the body of the faculty to react to the infection, either temporarily or for good.

So the result of our research done at Saigon showed that leprosy has a more serious development in the male sex. Moreover, it seems that puberty brings about an appreciable increase in the number of malignant cases among boys while it diminishes the risk of malignant development among girls. This resistance of the female body to the bacillary dissemination and invasion gives way in a marked manner between 20 and 30 years, an active period concerned with gestation. Clinical observations indicate moreover that pregnancy and confinement often cause an increase in the development of leprosy. Besides, this resistance to infection weakens among the Vietnamese and the Chinese between 40 and 50 years, that is to say during the menopause. In our opinion, the original anergy and the loss of the immunoallergic state in leprosy could be the result of modifications in the metabolism, partly determined by physiological changes or by pathological disturbances of the endocrine secretions ${ }^{4}, 5$.

The immunological and prognostic value of the Mitsuda reaction owing to the previous attack on the body by the leprosy bacillus remains indisputable. However, it is recognised now that sensitivity to lepromin can be induced, not only by a primary leprosy infection but also by tuberculosis or vaccination by BCG and also, according to certain authors, by repeated intradermal injection of lepromin, that is to say of heat-killed Hansen's bacilli. In these cases, may the Mitsuda reaction be considered as the sign of an immuno-allergic state equal in value to that determined by a primary leprosy infection? It is impossible to reply to this question with certainty.

As regards tuberculosis, a mycobacterial infection related to leprosy as the haemagglutin ation of Middlebrook-Dubos ${ }^{9.18}$ showed, we are convinced that a Mitsuda reaction, due to the impregnation or to the infection by the Koch bacillus, is equally the sign of a certain state of resistance in the body to the Hansen's bacillus. All the leprologists who have studied during epidemiological research the theory of antagonism between tuberculosis and leprosy which we put forward in $1944^{1}$ have come to conclusions in favour of this 
theory. In effect, leprosy disappears gradually wherever tuberculosis greatly spreads and it can only flourish in those parts little or recently attacked by tuberculosis. But has this para-allergy brought about by the Koch bacillus the same immunological significance as the allergy released by a leprosy infection? In fact we do not know. It is however possible that the eventual relative immunity caused by the tuberculosis infection is more feeble than that produced by the pathogenic agent of leprosy, since the Koch bacillus is an acid-resistant germ of a similar kind, yet different even so from the Hansen's bacillus. New epidemiological, clinical, and immunological studies will doubtless bring light on this complex subject.

Vaccination by BCG determines in a very large percentage the conversion of the Mitsuda reaction among people free from leprosy and tuberculosis. For, if tuberculosis brings about a certain state of defence in the body against leprosy, vaccination by BCG should have the same effect, but seemingly to a lesser degree, BCG being a germ of bovine origin and of very small virulence which can only maintain itself in a body for a relatively short time?

If it should come about that the para-allergy set up by BCG was really the sign of a certain state of resistance to the Hansen's bacillus, the problem of the prophylaxis of leprosy would be very largely solved. But although numerous publications on this subject have given favourable results, new experiments should be undertaken in the course of which we shall see how the control group not vaccinated compares with the vaccinated group as regards exposure to the infection, age, sex, and notably the previous clinical examination. Moreover, periodic revaccinations seem essential as occurs in premunition against tuberculosis.

It seems to us that the following experiment, carried out with children resistant to lepromin and to strong doses of tuberculin, could furnish useful indications of the eventual preventive action of BCG against leprosy.

Groups of controls and of those to be vaccinated would be formed according to the recommendations given above. Children to be vaccinated would receive $100 \mathrm{mg}$. of BCG orally and would be revaccinated every year by the same method. Finally, those vaccinated and the controls would undergo every year a thorough clinical examination.

The interpretation of the nature of the Mitsuda reaction obtained after repeated injections of lepromin is very tricky. One must at once state that trials in man have been carried out in a way open to criticism. In effect, this experiment had been carried out in the first place on the children of leprosy patients and then on people apparently healthy, living in regions where leprosy and tuberculosis were more or less endemic ${ }^{10,11}$. It is therefore impossible to know if certain of these Mitsuda reactions had been provoked by 
the repeated injection of lepromin or by unknown latent infections due to Hansen's bacillus or Koch's bacillus. It would seem better to carry out this experiment in countries where leprosy has been unknown for many years. The subjects of this research should be insensitive not only to lepromin but also to strong doses of tuberculin. Moreover, these people should be chosen from regions not exposed to the tuberculosis infection. Finally, among those who become sensitive to lepromin only those remaining negative to strong doses of tuberculin should be taken into consideration.

Can we claim, at least theoretically, that in the absence of all leprosy or tuberculosis infection the repeated injection of lepromin can assure a certain state of resistance to Hansen's bacillus? The study of immuno-allergy in tuberculosis could throw some light on the subject.

Thus, in a guinea-pig, after a subcutaneous injection of $0.1 \mathrm{mg}$. of virulent Koch bacillus, the superinfections produce after about 10 days ulcerated lesions of developing tuberculosis. However, as early as the appearance of sensitivity to tuberculin, which takes place about the 25th day, lesions of reinfection appear at shorter and shorter intervals. About 40 days after the primary inoculation if the animal is superinfected intradermally with a sufficient dose of bacilli the phenomenon of Koch appears.

In fact, one or two days after this superinfection one notes the formation of a necrosing ecchymotic infiltration at the point of injection. The injected bacilli are generally eliminated with the scab, and there is no glandular lesion. The superinfection is arrested while the primary infection follows its normal fatal evolution. The Koch phenomenon consists of two superimposed reactions, one of hypersensitivity characterised by the ecchymotic and necrotic changes, the other of immunity, expressed by the arrest of the tuberculosis superinfection.

Hypersensitivity can be discovered and even provoked just as well with living bacilli as with dead bacilli. There is an antigenic sensitivity, analogous to tuberculin sensitivity. On the contrary, the state of resistance to superinfections, or premunition can only be obtained after a primary infection, that is to say after an inoculation of living Koch bacilli. So the normal guinea-pig, previously injected with dead bacilli, will become allergic to tuberculin and present a typical Koch phenomenon after a second injection of dead bacilli, given about 40 days later. However, in spite of the indisputable Koch phenomenon, the animal will not be immune since an inoculation of living bacilli will produce developing tuberculosis. It appears then from one point of view that the allergic test is not the sign of a state of immunity but only of an acquired sensitivity, and from another that the immunity of superinfection or premunition can only be induced by the presence of living bacilli in the body. More- 
over, if the superinfection is carried out at a feeble dosage, only the phenomenon of resistance will appear. A state refractory to superinfection can therefore be produced without being preceded by local or general signs of hypersensitivity.

It is possible to conclude that allergy and immunity represent two reactional capacities, which, even if they are habitually joined and superimposed, remain separate, whether in spontaneous conditions of infectious pathology or in experiment (Gastinel).

It should also be stated that in 1944 we brought about for tuberculosis a reaction similar to the Mitsuda one, from which came the BCG test ${ }^{2 .}$ 3. ${ }^{6}$. The antigen was composed of a suspension of virulent Koch bacilli, heat-killed, in $0.5 \%$ carbolised physiological saline. The number of germs in the antigen was approximately equal to the number of Hansen's bacilli contained in lepromin. We were able to show with tuberculous patients that the reaction provoked by that antigen was macroscopically and histologically similar to that of Mitsuda. Moreover, the phenomena of hypersensitivity were more marked and their evolution was more rapid. In effect, the height of the reaction, which showed itself by ulceration, was obtained as early as at 1 or 2 weeks.

Now we had to prove on guinea-pigs that this reaction similar to that of Mitsuda in leprosy was not always accompanied by an immune state, since animals inoculated with dead bacilli and sensitized to this reaction, died after a later inoculation of virulent Koch bacilli. On the contrary, the guinea-pigs with a positive reaction after a primary infection of tuberculosis resisted superinfections. In tuberculosis the reaction to the antigen prepared with heat-killed Koch bacilli is only, like the Koch phenomenon, a hypersensitivity reaction. It only acquires an immunological significance in a body previously impregnated with living germs.

The nature of the sensitivity to lepromin of a body impregnated or infected by Hansen's bacillus seems to be close to that of the Koch phenomenon and to the reaction that we have suggested for tuberculosis. In effect, the Mitsuda reaction is equally shown by a hypersensitivity, accompanied by an immune state. However, the signs of hypersensitivity are less pronounced, while the relative immunity shows itself more powerful since it hinders the malignant evolution of the primary infection. Nevertheless, this immunoallergy can diminish in intensity and even disappear completely. Moreover, the Mitsuda reaction usually begins 1 or 2 days after the injection of the antigen (early reaction of Fernandez), but its final development is less acute and of longer duration. Finally, to the leprominic allergy properly so called is joined a foreign body reaction. The antigen contains in fact tissue debris and Lopes de Faria showed in 1953 that an injection of $0.1 \mathrm{ml}$. of a suspension of normal cutaneous tissue, crushed and filtered, in physiological saline 
(1:20) could provoke a weak reaction, but macroscopically and histologically identical to that of Mitsuda' ${ }^{12}$, in lepromin sensitive subjects. It is also known that the bacillary antigen of Fernandez, which no longer contains tissue debris, in practice gives late Mitsuda reactions clearly more feeble than lepromin prepared according to the Hayashi-Wade technique.

It appears from the study of immuno-allergy in tuberculosis, a mycobacterial infection related to leprosy, that it is very unlikely that the repeated injection of lepromin, that is to say of dead Hansen's bacilli, could bring about the appearance of relative immunity. This proceeding could more or less determine a hypersensitivity, bound to a foreign body reaction. Besides, the clinical and typical histological aspect of a Mitsuda reaction is not always witness of an immune state.

Thus, Convit, Lowe, and their collaborators could note that intradermal BCG vaccination brought about in 11 to $41 \%$ of lepromatous patients a slight sensitivity to lepromin ${ }^{8},{ }^{13}$. Now it seems improbable that this sensitivity could be interpreted as the sign of an immune state. One will hardly believe that a BCG injection can cause such an immunological change in the body of patients very strongly bacilliferous and anergic to the Hansen's bacillus, since one knows that a lepromatous patient, ulteriorly impregnated or infected by the Koch's bacillus, always remains insensitive to lepromin. Lowe has also shown that these weakly positive Mitsuda reactions noted in lepromatous cases vaccinated with BCG are of short duration and are not followed by clinical or bacteriological improvement in spite of continued treatment. It can only be in these cases a transitory cutaneous sensitivity without immunological significance ${ }^{5}$.

From this it seems that sensitivity to lepromin can be considered as an allergic phenomenon, linked to a foreign body reaction. It will not be ipso facto witness of an immune state and will only assume an immunological value with subjects already impregnated by living Hansen's bacilli. The present theory, which is to interpret the early reaction of Fernandez as a phenomenon of hypersensitivity and the late Mitsuda reaction as a test of immunity, will have to be revised.

Tuberculosis and leprosy are related mycobacterial infections and it is therefore possible that the para-allergy to the Hansen's bacillus of subjects attacked by tuberculosis or BCG vaccinated is also bound up with a certain degree of resistance in the body to the leprosy infection. However, only clinical observation will finally give us grounds to affirm this.

As regards the allergic Mitsuda reaction, provoked by the repeated injection of lepromin, it is apparent that it only represents a cutaneous sensitivity without immunological significance. 


\section{Summary}

The author attempts to define the nature and significance of the Mitsuda reaction by studying the clinical and immunological data on leprosy and tuberculosis, which are related mycobacterial infections, as evidenced by the Middlebrook-Dubos haemagglutination reaction.

The author considers sensitivity to lepromin as an allergic phenomenon, associated with a foreign body reaction. This sensitivity does not necessarily prove immunity, and should only have immunological significance in individuals already contaminated by living Hansen bacilli. The current theory, considering the early Fernandez reaction as a sign of sensitization and the late Mitsuda reaction as an immunity test, should then be revised. However, the paraallergy to Hansen bacilli observed in tuberculous or BCG-vaccinated individuals might be related to a certain degree of resistance to leprosy infection: Only clinical study will allow a conclusion.

Mitsuda allergic reaction induced by repeated injections of lepromin (i.e. dead Hansen bacilli) seems to be due exclusively to cutaneous sensitization without immunological significance.

\section{References}

1. Chaussinand, R., Rev. Med. Franc. E.-O., 1944, 22, 667; Int. J. Lep., 1948, 16, 431 and $1957,25,365$.

2. Chaussinand, R., Rev. Med. Franc. E.-O., 1944, 22, 799; Ann. Inst. Pastelli, 1947, 73, 811 .

3. Chaussinand, R., Rev. Med. Franc. E.-O., 1944, 22, 803; Ann. Inst. Pastelur, 1947, 73, 814.

4. Chaussinand, R., Int. J. Lep., 1947, 15, 406.

5. Chaussinand, R., La Lèpre, $2 e$ edit. Expansion scientifique française, Paris, 1955.

6. Chaussinand, R., Rev. Tub., 1957, 21, 530.

7. Chaussinand, R. (in collaboration with M. Viette). Prophylaxie et thérapeutique de la lepre, G. Doin, Paris, 1958.

8. Convit, J., Rossi, E., Rodriguez, F. C., and Contreras, R., Int. J. Lep., 1952, 20, 347.

9. Gernez-Rifux, C., Montestruc, E., and Tacquet, A., Bull. Acad. Nat. Med., 1952, 136, 375.

10. Ignacio, J. L., Palafox, C. A., and Jose, F. A., Jr., Int. J. Lep., 1955, 23, 259.

11. Lara, C. B., Month. Bull. Bur. Hlth. Manila, 1939, 19, 15; Int. J. Lep., 1940, 8,15 .

12. LOPES DE FARIA, J., Contribuiçâo ao conhecimento da natureza da reaçâo de Mitsuda. Departamento de Imprensa Nacional, Rio de Janeiro, 1953.

13. Lowe, J., and MaCNULTY, Int. J. Lep., 1953, 21, 173.

14. Pessôa Mendes, J. Tese de concurso à Livre-Docência, 1956, Pôrto Alegre.

15. Souza Campos, N., Rev. Bras. Lep., 1937, 5, 99.

16. Souza-Lima, L. DE, and Souza Campos, N. Lepra tuberculoide, Editora Renascença, Sâo-Paulo, 1947.

17. Rotberg, A., VIe Congrès, Int. Lep., 1953, 656.

18. Viette, M., Ann. Inst. Pasteur, 1954, 86, 76. 